\title{
Congenital dysfibrinogenemia as a rare cause of recurrent gastrointestinal bleeding
}

\author{
Weronika Lebowa', Jakub Kenig', Joanna Zdziarska ${ }^{2}$ \\ 'Department of Ceneral, Oncologic and Ceriatric Surgery, Jagiellonian University Medical College, Krakow, Poland \\ ${ }^{2}$ Department of Hematology, Jagiellonian University Medical College, Krakow, Poland; Head: prof. Tomasz Sacha MD PhD
}

Article history: Received: 06.03.2020 Accepted: 09.04.2020 Published: 16.04 .2020

ABSTRACT: Introduction: Gastrointestinal bleeding is a common disease that surgeons encounter in everyday clinical practice. It is most often easy to diagnose and treat. However, rare causes of bleeding can lead to delayed diagnosis and ineffective treatment. Dysfibrinogenemia is a qualitative fibrinogen disorder in which functional fibrinogen level is reduced with normal antigenic level.

Case report: Herein we present the case of a 59-year-old female with recurrent gastrointestinal bleeds, that turned out to be an unusual manifestation of congenital dysfibrinogenemia. Detailed imaging and endoscopic diagnostics revealed portal hypertension with a non-bleeding $1-\mathrm{cm}$ gastrointestinal stromal tumor and multiple angiodysplastic lesions in close proximity.

\section{ABBREVIATIONS}

angio-CT - computed tomography of blood vessels

FFP - fresh frozen plasma

GIST - gastrointestinal stromal tumor

Hgb - hemoglobin

HHT - hereditary hemorrhagic telangiectasia

PLT - thrombocytes

WBC - white blood cells

\section{INTRODUCTION}

Fibrinogen is a glycoprotein synthesized and secreted into the blood primarily by liver hepatocyte cells. Fibrinogen is the most abundant clotting factor, with usual blood concentrations ranging from 1.8 to $3.5 \mathrm{~g} / \mathrm{L}$. It also binds to platelets, supports aggregation, and plays an important role in wound healing $[1,2]$. Congenital dysfibrinogenemia is a rare hereditary disease characterized by normal antigen level but lower function level of fibrinogen. Clinical manifestation includes bleeding or thrombotic events, or both simultaneously [3]. However, in many cases dysfibrinogenemia is asymptomatic or has a nonspecific clinical picture, sometimes patients present anemia secondary to recurrent occult gastrointestinal bleeding [4].The aim of this study was to present the case of a 59-year-old female with recurrent gastrointestinal bleeding caused by dysfibrinogenemia.

\section{CASE PRESENTATION}

A 59-year-old female was admitted to the Department of General Surgery after an upper gastrointestinal bleeding episode. On admission she complained of exercise tolerance deterioration, dizziness and general malaise lasting few days, without stenocardial complaints or bleeding symptoms. The patient's physical examination was generally unremarkable except for skin pallor. No bleeding on the skin or the mucosa was observed, previous teeth extractions, delivery and breast biopsy caused non-hemorrhagic complications. Family history, however, was remarkable: her father and daughter suffered from lifelong, spontaneous nose bleeds (however, this was revealed during further investigation). She underwent gastroscopy revealing a small gastric ulceration on the top of an intramural tumor and angiodysplastic mucosal lesions in its area. Histopathological tests showed chronic gastritis with the presence of Helicobacter pylori. Computed tomography confirmed the presence of gastric tumor with a diameter of $10 \mathrm{~mm}$ suggestive of gastrointestinal tumor (GIST). A surgical treatment was considered suspecting that the tumor may be the cause of the bleeding. However, preoperative diagnostics revealed portal hypertension in the abdominal angio-CT. Diagnosis was supplemented with colonoscopy and capsule endoscopy which were without any pathology. Later she was hospitalized several times in the Department of Internal Medicine due to recurrent episodes of severe anemia (every 6-8 weeks) and treated with packed red blood cells transfusions. Gastroscopy was also repeated several times, revealing the same results, without any active or past bleeding. Laboratory testing showed severe anemia (HGB level $7.3 \mathrm{~g} / \mathrm{dL}$ ), moderate leukopenia (WBC count of 2600/uL) and thrombocytopenia (PLT count of $126000 / \mathrm{uL}$ ). Liver parameters were normal. Due to the clinical picture, hematological consultation was ordered. The patient was diagnosed with dysfibrinogenemia. Clottable fibrinogen concentration estimated with von Clauss method was $1.4 \mathrm{~g} / \mathrm{L}$ (normal range 1.8-3.5 g/L) and fibrinogen antigen level determined nephelometrically: $3.8 \mathrm{~g} / \mathrm{L}$ (normal range $1.8-3.5 \mathrm{~g} / \mathrm{L}$ ). Due to tardive clinical symptoms onset and concomitantly diagnosed portal hypertension, acquired dysfibrinogenemia was considered first. However, fibrinogen concentration measured in the patient's daughter was also diagnostic for dysfibrinogenemia $(1.0 \mathrm{~g} / \mathrm{L}$ with von Clauss method and $3.0 \mathrm{~g} / \mathrm{L}$ with nephelometry), which confirms the congenital character of this coagulation disorder. Since the patient was ordered to take tranexamic acid at a dose of $1000 \mathrm{mg}$ three times a day, she suffered from only one gastrointestinal bleed that was treated with cryoprecipitate. She is now being observed in the hemophilia treatment center with the intention to implement prophylaxis with fibrinogen concentrate, if clinically indicated. Iron supplementation led to resolution of anemia.

\section{DISCUSSION}

Patients with dysfibrinogenemia have an unpredictable clinical phenotype. The majority of them are asymptomatic and diagnosed incidentally. Clinical manifestations include venous (or rarely 
arterial) thrombosis (approximately $20 \%$ of patients) or bleeding, occurring during invasive procedures (e.g. tooth extraction) or spontaneously, like epistaxis (25\% of patients) $[5,6]$. The prevalence of dysfibrinogenemia is estimated at 1-2 per million, however it is difficult to establish because of the large number of asymptomatic cases [7]. The diagnosis is suspected from prolonged coagulation times, with low fibrinogen measured by functional assay. Normal or high antigen levels and low functional activity are typical, with the ratio of functionally-detected to immunologicallydetected fibrinogen concentration usually below 0.7 [2]. Partial thromboplastin time, activated partial thromboplastin time, thrombin time, and reptilase time tests are usually prolonged, regardless of history of bleeding or thrombosis [3].

In our patient, the diagnosis of dysfibrinogenemia was made only after specialist hematological consultation. A gastric tumor suspected of being the site of bleeding and recurrent episodes of anemia as a chief complaint complicated the diagnostic process. Initially, our patient was qualified for laparoscopic wedge-shaped gastrectomy, but unexplained, gastrointestinal bleeds were considered to indicate high perioperative risk of uncontrolled hemorrhage. In fact, surgery would probably have caused hemorrhage, as the diagnosis of the bleeding disorder was not known at that time.

Menorrhagia and easy bruising are the most frequent bleeding incidences in patients affected with dysfibrinogenemia. Gastrointestinal bleeding has also been reported, although much less frequently $[5,8]$. Wypasek et al. reported that only $3.7 \%$ of patients developed gastrointestinal bleeding [8]. Another study presented a similar incidence of these $-3.5 \%$ [9]. Gastrointestinal bleeding as the only symptom of dysfibrinogenemia, like in the presented patient, poses diagnostic difficulties, because the main reasons for intestinal hemorrhages include detectable organic lesions in the digestive tract [4]. In general, in case of suspected gastrointestinal bleeding the first step is endoscopic examinations focused on bleeding site localization, especially duodenal and gastric ulcers. According to the diagnostic algorithm, the following tests are usually performed: two gastroscopies and two colonoscopies and capsule endoscopy (until the bleeding site is found). If negative, rarer reasons for chronic anaemia are searched for, including coagulopathy [10].

Dysfibrinogenemia may either be autosomal-dominantly inherited or acquired. Regarding congenital dysfibrinogenemia, more than 100 mutations and 400 families have been reported on [1]. Abnormal fibrinogen is found in patients with liver diseases characterized by increased sialic acid content. In the presented case, portal hypertension observed in the abdominal angio-CT was probably the result of portal vein thrombosis, secondary to hematological disease. However, acquired dysfibrinogenemia may also occur in other systemic diseases such as multiple myeloma, autoimmune disorders, malignancy, and with administration of some medications (glucocorticoids, isotretinoin, and antileukemic agents) $[9,11]$.

Congenital dysfibrinogenemia requires differentiation with other congenital hemorrhagic diatheses, especially with inherited von Willebrand disease and hereditary hemorrhagic telangiectasia, also known as Rendu-Osler-Weber syndrome. Iron-deficient anemia may be the main symptom of all these disease entities. The prevalence of angiodysplasia in inherited von Willebrand was $2 \%$ and $4.5 \%$ for types 2 and 3, respectively [12]. In case of HHT gastrointestinal telangiectasias are quite frequent (70\% of patients) and may lead to hemorrhages and anemia [13]. Pharmacological treatment is based on tranexamic acid administration and implementation of prophylaxis with deficient coagulation factor. New drugs could be effective in the treatment of gastrointestinal bleeding due to angiodysplasia: Thalidomide, Bevacizumab and Tacrolimus [13, 14]. Patients with a bleeding phenotype should be referred to a hemophilia treatment center for observation and management. In cases of bleeding, fresh frozen plasma (FFP), cryoprecipitate or fibrinogen concentrate can be administered, according to their availability [15]. In the case of acquired dysfibrinogenemia the underlying disease should also be treated.

\section{CONCLUSIONS}

The attendant physician must be alert to rare causes of bleeding, especially if the patient has simultaneously other organic reasons for bleeding. Recurrent idiopathic gastrointestinal bleeding remains a diagnostic challenge to the clinician and a constant threat to the patient. Although congenital bleeding disorder is a relatively rare cause of gastrointestinal bleeding, it should be ruled out in patients with chronic anaemia. Bleeds and surgeries in patients with fibrinogen deficiencies and other congenital coagulation defects should be managed with deficient clotting factor concentrates, in strict cooperation with a hemophilia treatment center.

\section{REFERENCES}

1. Castaman G., Giacomelli S.H., Biasoli C., Contino L., Radossi P.: Risk of Bleeding and Thrombosis inInherited Qualitative Fibrinogen Disorders. Eur J Haematol., 2019; 103(4): 379-384.

2. Caimia G., Rasoa S., Napolitanoa M., Hoppsa E., Lo Prestib R., Siragusa S.: Haemorheological profile in congenital afibrinogenemia and in congenital dysfibrinogenemia: A clinical case report. Clin Hemorheol Microcirc., 2019; 73(4): 523-530.

3. Neerman-Arbez M., de Moerloose P., Casini A.: Laboratory and Genetic Investigation of Mutations Accounting for Congenital Fibrinogen Disorders. Semin Thromb Hemost., 2016; 42(4): 356-365.

4. Toskes P.P., Wagger G.M., Raskin H.F., Attar S.: Coagulation Defects in Undiagnosed Recurrent Gastrointestinal Bleeding. Ann. Surg., 1970; 172(1): 98-108.

5. Shapiro S.E., Phillips E., Manning R.A., Morse C.V., Murden S.L., Laffan M.A. et al.: Clinical phenotype, laboratory features and genotype of 35 patients with heritable dysfibrinogenaemia. Br J Haematol., 2013; 160(2): 220-227.

6. Zhou J., Zhu P., Zhang X.: A Chinese family with congenital Dysfirinogenemia carries a heterozygous missense mutation in FGA: Concerning the genetic abnormality and clinical treatment. Pak J Med Sci., 2017; 33(4): 968-972.

7. Casini A., de Moerloose P., Neerman-Arbez M.: Clinical features and management of congenital firinogen deficiencies. Semin Thromb Hemost., 2016; 42(4): 366-374.

8. Wypasek E., Klukowska A., Zdziarska J. et al.: Genetic and clinical characterization of congenital firinogen disorders in Polish patients: Identifiation of three novel firinogen gamma chain mutations. Thromb Res., 2019; 182: 133-140.

9. Casini A., Neerman-Arbez M., Ariens R.A., de Moerloose P.: Dysfibrinogenemia: from molecular anomalies to clinical manifestations and management. J Thromb Haemost., 2015; 13(6): 909-919.

10. Kim B.S., Li B.T., Engel A., Samara J.S.: Diagnosis of gastrointestinal bleeding: A practical guide for clinicians. World J Gastrointest Pathophysiol., 2014; 5(4): 467-478.

11. Undas A., Zabczyk M., Iwaniec T.: Dysfibrinogenemia: from bleeding tendency to thromboembolic disorders. Boletim da SPHM, 2011; 26: 5-17. 
12. Morris E.S., Hampton K.K., Nesbitt I.M., Preston F.E., Thomas W.E.G., Makris M.: The management of von Willebrand's disease-associated gastrointestinal angiodysplasia. Blood Coagul Fibrinolysis., 2001; 12(2): 143-148.

13. Robert F., Desroches-Castan A., Bailly S., Dupuis-Girod S., Feige J.J.: Future treatments for hereditary hemorrhagic telangiectasia. Orphanet.J Rare Dis., 2020; 15(1): 4
14. Ou G., Galorport C., Enns R.: Bevacizumab and gastrointestinal bleeding in hereditary hemorrhagic telangiectasia. World J Gastrointest Sur., 2016; 8(12): 792-795

15. de Moerloose P., Neerman-Arbez M.: Treatment of congenital fibrinogen disorders. Expert Opin Biol Ther., 2008; 8(7): 979-992.

\begin{tabular}{|c|c|c|c|c|}
\hline Liczba słów: 1660 & Liczba stron: 3 & Tabele: - & Ryciny:- & Piśmiennictwo: 15 \\
\hline
\end{tabular}

DOI: $\quad 10.5604 / 01.3001 .0014 .0948$

Table of content: https://ppch.pl/resources/html/articlesList?issueld=0

Copyright: Copyright @ 2020 Fundacja Polski Przegląd Chirurgiczny. Published by Index Copernicus Sp. z o. o. All rights reserved.

Competing interests: The authors declare that they have no competing interests.

(2) The content of the journal "Polish Journal of Surgery" is circulated on the basis of the Open Access which means free and

- limitless access to scientific data.

This material is available under the Creative Commons - Attribution 4.0 GB. The full terms of this license are available on: http://creativecommons.org/licenses/by-nc-sa/4.0/legalcode

Corresponding author: Jakub Kenig (ORCID: 0000-0001-5323-4247); Department of General, Oncologic and Geriatric Surgery, Jagiellonian University Medical College, Krakow, Poland; Prądnicka Street 35-37, 31-202 Krakow, Poland;

E-mail: jkenig@cm-uj.krakow.pl

Cite this article as: Lebowa W., Kenig J., Zdziarska J.: Congenital dysfibrinogenemia as a rare cause of recurrent gastrointestinal bleeding; Pol Przegl Chir 2020: 92: 1-3; DOI: 10.5604/01.3001.0014.0948 (Advanced online publication) 